Check for updates

Cite this: Phys. Chem. Chem. Phys. 2020, 22, 12697

Received 8th May 2020,

Accepted 22nd May 2020

DOI: $10.1039 / \mathrm{d} 0 \mathrm{cp} 02513 \mathrm{e}$

rsc.li/pccp

\section{Predicting the phase diagram of titanium dioxide with random search and pattern recognition $\dagger$}

\begin{abstract}
Aleks Reinhardt, ${ }^{\mathrm{a}}$ Chris J. Pickard ${ }^{\mathrm{bc}}$ and Bingqing Cheng*ade
Predicting phase stabilities of crystal polymorphs is central to computational materials science and chemistry. Such predictions are challenging because they first require searching for potential energy minima and then performing arduous free-energy calculations to account for entropic effects at finite temperatures. Here, we develop a framework that facilitates such predictions by exploiting all the information obtained from random searches of crystal structures. This framework combines automated clustering, classification and visualisation of crystal structures with machine-learning estimation of their enthalpy and entropy. We demonstrate the framework on the technologically important system of $\mathrm{TiO}_{2}$, which has many polymorphs, without relying on prior knowledge of known phases. We find a number of new phases and predict the phase diagram and metastabilities of crystal polymorphs at $1600 \mathrm{~K}$, benchmarking the results against full free-energy calculations.
\end{abstract}

\section{Introduction}

Predicting the properties of solid materials requires an understanding of how atoms are arranged, which in turn necessitates the determination of the relative stabilities of possible crystal polymorphic phases under various conditions. Advances in crystal structure prediction, ${ }^{1}$ including random structure search (RSS), ${ }^{2}$ particle-swarm optimisation, ${ }^{3}$ Monte Carlo simulations with variable box shapes ${ }^{4}$ and basin hopping, ${ }^{5}$ allow us to find minima on the potential-energy surface (PES) and thus to identify potentially competitive polymorphs. However, in these approaches only the enthalpy $H$ at $0 \mathrm{~K}$ is typically computed and used to determine phase stability, even though the difference in entropy $S$ between polymorphs can play a significant role. ${ }^{6,7}$ Relative Gibbs energies $(G=H-T S)$, which in actuality dictate thermodynamic (meta)stability, are seldom computed, despite their importance for phases that can be synthesised at one thermodynamic condition but remain metastable under other conditions.

Although Gibbs energies can be computed using free-energy methods such as thermodynamic integration (TI), ${ }^{6,8,9}$ such

\footnotetext{
${ }^{a}$ Department of Chemistry, University of Cambridge, Lensfield Road, Cambridge, CB2 1EW, UK.E-mail: bc509@cam.ac.uk

${ }^{b}$ Department of Materials Science \& Metallurgy, University of Cambridge, 27 Charles Babbage Road, Cambridge, CB3 OFS, UK

${ }^{c}$ Advanced Institute for Materials Research, Tohoku University, Sendai, Japan

${ }^{d}$ TCM Group, Cavendish Laboratory, University of Cambridge, J. J. Thomson Avenue, Cambridge, CB3 OHE, UK

${ }^{e}$ Trinity College, Trinity Street, Cambridge, CB2 1TQ, UK

$\dagger$ Electronic supplementary information (ESI) available. See DOI: 10.1039/ docp02513e
}

calculations are non-trivial and usually require long simulations, making them both expensive and tedious if one wants to use the PES computed from first-principles methods such as density-functional theory (DFT). ${ }^{10}$ Moreover, crystal-structure prediction schemes typically result in a multitude of structures. It can be challenging to rationalise how the structures are related to one another and which ones are promising candidates under given thermodynamic conditions.

In this study, we propose an approximate method that combines RSS and machine learning (ML) to estimate free energies of solid phases and hence the thermodynamic phase behaviour of a system of interest with relatively little computational effort and without relying on prior knowledge of the known phases. We have selected titanium dioxide at ambient and high pressures to benchmark the method because it is a widely used metal oxide with a large number of polymorphs (see the ESI $\dagger$ ), many of which may have interesting optical, ${ }^{11}$ mechanical $^{12}$ and electrochemical ${ }^{13}$ properties. Furthermore, high-pressure $\mathrm{TiO}_{2}$ phases can also serve as analogues of the structures adopted by many other important $\mathrm{AX}_{2}$ systems that are of particular interest in geology, such as high-pressure silicas. ${ }^{14}$

\section{Methods}

\section{A. Calculations using empirical potential}

For RSS and accurate free energy calculations, we focus on the simple MA empirical pair potential for $\mathrm{TiO}_{2}{ }^{15}$ because of its low computational cost. ${ }^{9}$

We performed RSS at $0 \mathrm{GPa}, 20 \mathrm{GPa}, 40 \mathrm{GPa}$ and $60 \mathrm{GPa}$ using the AIRSS ${ }^{16,17}$ package interfaced with Lammps. ${ }^{18}$ For each RSS 
run, we first chose a reasonable cell shape at random, and added 2, 3, 4, 6, 8, 9 or 12 formula units $\mathrm{TiO}_{2}$ of into the simulation cell at random positions while keeping the initial density of the cell close to the typical density range of this system. We set a lower bound on the interatomic distance for each pair of atomic species, but otherwise imposed no additional constraints or symmetries on the initial structures. We then relaxed each structure using a second-order conjugate gradient algorithm until the forces on atoms and the difference between the target and actual pressures both became negligible.

To benchmark how well our approximate framework can predict the phase behaviour $\mathrm{TiO}_{2}$ of at ambient and high pressures as described by the MA potential, we also performed free-energy calculations for both the known phases and the new phases obtained from RSS. Specifically, we computed the chemical potentials of the polymorphs using Frenkel-Ladd integration ${ }^{8,19}$ and subsequent TI along isobars and isotherms, as detailed in ref. 9.

All the necessary input files for performing the abovementioned calculations using AIRSS and Lammps are provided in the ESI. $\dagger$

\section{B. DFT calculations}

For the metastable polymorphs identified with the MA potential, we performed geometrical optimisation and computed the enthalpies at the DFT level over a pressure range of $0 \mathrm{GPa}$ to $70 \mathrm{GPa}$ in steps of $10 \mathrm{GPa}$. We separately employed three common functionals, $\mathrm{LDA}^{20}{ }^{20} \mathrm{PBE}^{21}$ and PBEsol, ${ }^{22}$ using the CASTEP $a b$ initio simulation package. ${ }^{23}$ Full details of the DFT set-ups and configurations can be found in the input files supplied in the ESI. $\dagger$

The key value of the current study lies in the data analysis, which we describe in the following section. A Python notebook implementing the machine learning and data processing steps, input and data files are available on the public repository https://github.com/BingqingCheng/TiO2_random_search_pattern_ recognition.

\section{Results and analysis}

\section{A. Characterisation of structures found by RSS}

At each pressure, RSS using the MA potential produced thousands of distinct $\mathrm{TiO}_{2}$ structures with different atomic co-ordinates, cell shapes and numbers of formula units in the cell. Even though knowledge of the space groups, molar volumes and energies of the structures provides hints on how to classify them, it is still a formidable task to sort through them manually. In recent years, ML-inspired approaches have been used for the classification and visualisation of atomic structures, ${ }^{24-28}$ but they have not been systematically exploited in the context of recognising the metastable polymorphs from RSS, especially for the purpose of determining the phase behaviour of crystalline systems. We have therefore developed and employed a ML-based method to compare and cluster the structures automatically.

a. Similarity measurements. This pattern recognition task is built around the construction of a kernel matrix $\{K(A, B)\}$ that measures the similarity between each pair of structures $A$ and $B$ in the data set. The kernel matrix should be positive-definite and normalised. If it is not already normalised by construction, then $K(A, B)$ should be divided by $\sqrt{K(A, A) K(B, B)}$. The kernel function $K(A, B)$ can be formulated as an inner product of the features of $A$ and $B$,

$$
K(A, B)=\Phi(A)^{\mathrm{T}} \Phi(B)=\sum_{i=1}^{M} \phi_{i}(A) \phi_{i}(B),
$$

where $\Phi=\left\{\phi_{i}\right\}$ denotes the set of global fingerprints associated with the whole structure, rather than with individual atomic environments $\mathcal{X}_{n}^{A}$ that are centred on each atom $n$ in the structure $A$. A straightforward way of obtaining these global fingerprints from the local ones is to take the average, ${ }^{26}$

$$
\Phi(A)=\frac{1}{N_{\mathrm{A}}} \sum_{n=1}^{N_{\mathrm{A}}} \Psi\left(\mathcal{X}_{n}^{A}\right)
$$

where $N_{\mathrm{A}}$ denotes the total number of atoms in the system $A$ if it only contains one element. If the system contains multiple atomic species and if the local fingerprints are specific to atomic species, the average in eqn (2) should be taken over atoms of the same atomic species $\alpha$ and the resulting vectors associated with each species are then concatenated. Apart from the average kernel used here, one may also use the MATCH or RE-MATCH kernel of ref. 26, although we did not notice a significant improvement during the subsequent analysis.

We employ the Smooth Overlap of Atomic Positions (SOAP) framework that was introduced in ref. 29 to construct the local fingerprints $\Psi(\mathcal{X})$. SOAP has been used together with Gaussian Process Regression in numerous applications, including metals, semiconductors, molecular crystals and small organic molecules, ${ }^{26,30-33}$ as well for structural identifications of bulk materials, including ice ${ }^{25,27}$ and $\mathrm{TiO}_{2} \cdot{ }^{28}$ The SOAP representation is specific to atom species. For atoms of species $\alpha$ inside a local environment $\mathcal{X}$, it uses a smooth atomic density function

$$
\rho_{\mathcal{X}}^{\alpha}(\boldsymbol{r})=\sum_{i \in \mathcal{X}_{\alpha}} \exp \left(-\frac{\left[\boldsymbol{r}-\boldsymbol{r}_{i}\right]^{2}}{2 \sigma^{2}}\right)
$$

by summing over Gaussians centred on each atom $i$ of species $\alpha$ that has a displacement $\boldsymbol{r}_{i}$ within a given cutoff $r_{\mathrm{c}}$ of the central atom of the environment $\mathcal{X}$. The density $\rho_{\mathcal{X}}^{\alpha}(\boldsymbol{r})$ is invariant to translations and permutations of identical atoms, but not to rotations. The SOAP representation addresses this by first expanding with a set of orthonormal basis functions on radial direction $g(|r|)$ and spherical harmonics of angular directions $\hat{r}$ as

$$
\rho_{\mathcal{X}}^{\alpha}(\boldsymbol{r})=\sum_{n l m} c_{n l m}^{\alpha} g_{n}(|r|) Y_{l m}(\hat{r}),
$$

and then taking the power spectra that characterise the rotational-invariant arrangement of atoms of species $\alpha$ inside the local environment $\mathcal{X}^{26,29}$

$$
k_{n n^{\prime} l}^{\alpha}(\mathcal{X})=\pi \sqrt{\frac{8}{2 l+1}} \sum_{m}\left(c_{n l m}^{\alpha}\right)^{*} c_{n^{\prime} l m}^{\alpha} .
$$


The vector $\left\{k_{n n^{\prime} l}^{\alpha}\right\}$ constructed in this way up to certain cutoffs $l_{\text {max }}$ and $n_{\text {max }}$ can then be used as the local fingerprint $\Psi(\mathcal{X})$ in eqn (2), which in turn leads to the kernel matrix. In practice, we use the recently implemented DScribe Python package for constructing descriptors and kernels. ${ }^{34}$

b. Low-dimensional maps. The kernel matrix $\{K(A, B)\}$ provides distance measurements between structures in a highdimensional space. To visualise such distances, we project them onto a two-dimensional map using kernel principal component analysis (KPCA), ${ }^{35}$ which amounts to projecting $\{K(A, B)\}$ onto its two eigenvectors with the largest eigenvalues. Fig. 1 shows such $2 \mathrm{D}$ maps for the structures found during the RSS at $20 \mathrm{GPa}$.

c. Clustering. For the set of structures generated by RSS at each pressure, we clustered them based on the similarity measurements $\{K(A, B)\}$ in order to find which structures belong to the same crystallographic family using the Density-Based Spatial Clustering of Applications with Noise (DBSCAN) algorithm. ${ }^{36}$ In this approach, the density of data points around a given data point is first estimated and then points with a density above a certain threshold are identified as clusters. Input data points that are not ascribed to regions of high data density are classified as noise. The most important DBSCAN parameter is the maximum distance between two samples for them to be considered neighbours. In our case, there is a very clear separation between similar and dissimilar structures obtained from RSS due to the absence of thermal noise, so the clustering outcome is insensitive to this DBSCAN parameter.
As an example, in Fig. 1 we show such clustering results. Even though the information on space groups, molar volume and enthalpy is not explicitly included in the construction of the kernel matrix $\{K(A, B)\}$, this agnostic pattern recognition approach is able to group together structures with similar properties extremely well.

d. Identification of known and new phases. In the KPCA map of Fig. 1, we also project the location of previously found crystal structures of $\mathrm{TiO}_{2}$, and, in an ad hoc manner, the new structures found in the present study. If a cluster that contains several structures found in RSS also includes a previously known phase, it is given the label of this phase, otherwise it is regarded as a new phase. In this way, we found 15 phases which have not to our knowledge previously been considered in studies of titanium dioxide in this pressure range. In the remainder of this manuscript we label them with a standard format $P$-a-SYM, where $P$ gives the pressure in gigapascals at which the structure was found in RSS, a is a lowercase letter purely used for labelling, and SYM gives the space group of the structure. These new structures are illustrated and described in the ESI $\dagger$ (Fig. S1). One of the new phases $(60-\mathrm{d}-P \overline{6} 2 m)$ is in fact the $\mathrm{Fe}_{2} \mathrm{P}$ phase of $\mathrm{TiO}_{2}$ that was previously considered at higher pressures than we have focused on here. ${ }^{11,37}$

e. DFT calculations. Although the MA potential satisfactorily predicts many properties of $\mathrm{TiO}_{2},{ }^{38,39}$ it does not give a perfect description of interactions, so the fact that a new phase is found using the MA potential does not necessarily mean that the same holds for $\mathrm{TiO}_{2}$ in experiment. To check whether the new structures may be of experimental relevance, we computed

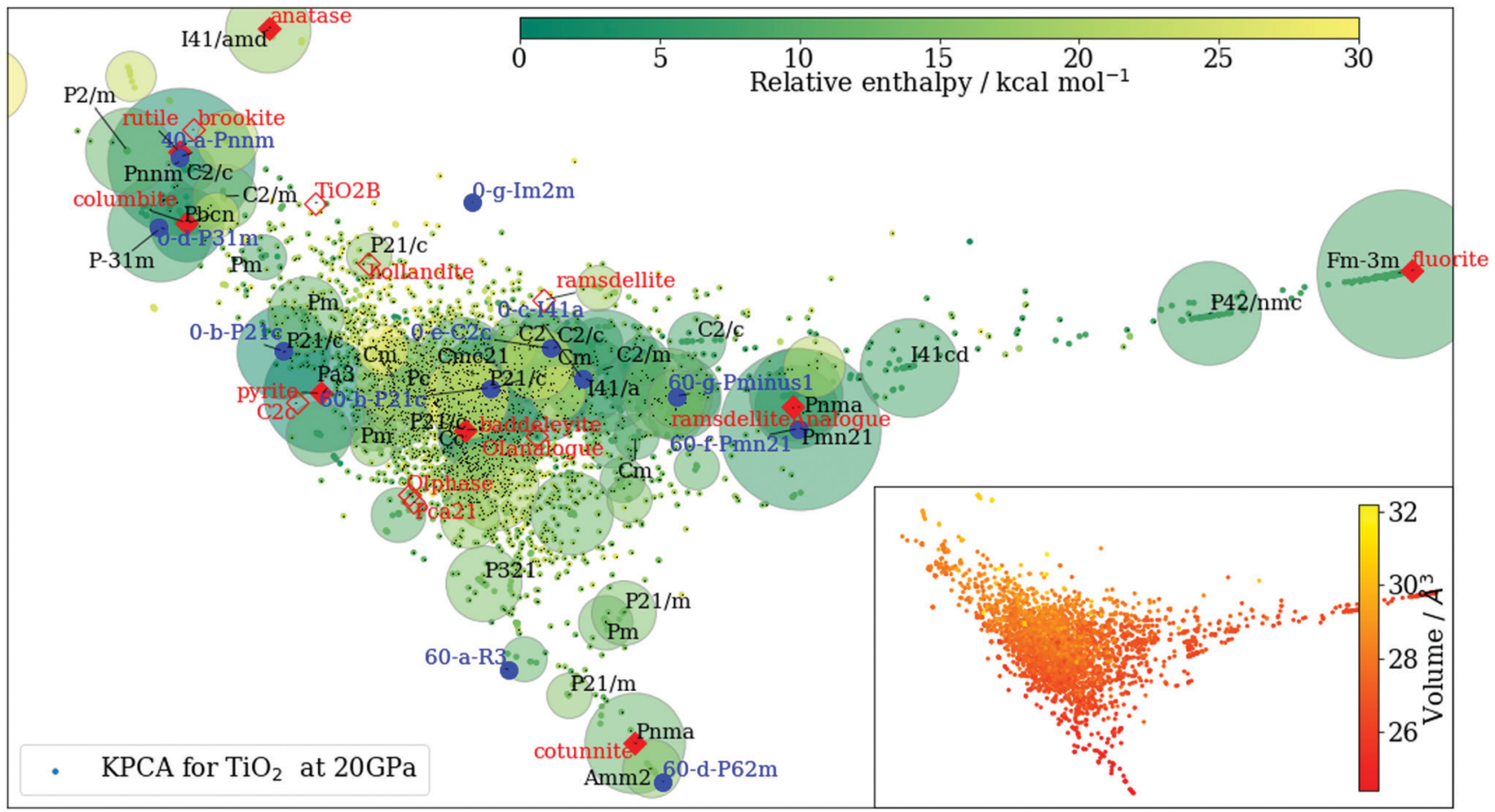

Fig. 1 Output after KPCA and clustering analysis. The structures obtained from RSS at $20 \mathrm{GPa}$ are projected onto the first two principal vectors of the kernel matrix $\{K(A, B)\}$. The known and new phases of $\mathrm{TiO}_{2}$ are indicated on the KPCA plot using solid markers if found during RSS, and hollow markers otherwise. The colours of the points in the main graph and the inset vary according to their enthalpy and volume per $\mathrm{TiO}_{2}$ formula unit, respectively. The area of each marker is proportional to the logarithm of the number of the structures in that cluster. 
the lattice energies and enthalpies of all the phases at the DFT level over a pressure range of $0 \mathrm{GPa}$ to $70 \mathrm{GPa}$ (see Methods). We used three functionals, LDA, PBE and PBEsol, and the results are shown in the ESI $\dagger$ (Fig. S3). Although a recent study $^{40}$ shows good agreement in the ranking of static-lattice energies of phases at low pressure between diffusion quantum Monte Carlo and a number of DFT functionals, the DFT calculations for $\mathrm{TiO}_{2}$ should be approached with a degree of caution particularly at low pressure, ${ }^{37}$ since for example rutile is not predicted to be the stable phase at sufficiently low pressures for any of them. The results for the known phases are consistent with previous work. ${ }^{7,41-43}$ At high pressures ( $\left.60 \mathrm{GPa}\right)$, the three functionals gave consistent results, suggesting that the results are robust under such conditions. Although none of the newly reported phases have the lowest enthalpy, several phases are very competitive. In particular, $60-\mathrm{d}-P \overline{6} 2 m$ and $60-\mathrm{e}-P 2_{1} / m$ have enthalpies very close to the ground-state phase of cotunnite at high pressures ( $P \geq 40 \mathrm{GPa}$ ), and the $60-\mathrm{a}-R \overline{3}$ phase, which, as we discuss below, is particularly favoured for the MA potential, has an enthalpy only a few kcal $\mathrm{mol}^{-1}$ per formula unit larger than cotunnite. The difference in relative enthalpy between different levels of theory highlights the importance of capturing metastable phases, as in general the enthalpies predicted by empirical potentials or density-functional approximations may not be accurate enough to determine the true ground-state enthalpy.

\section{B. Approximation of entropy using frequency of appearance}

In this section, we investigate whether it is possible to approximate the entropies of different phases solely from the information one can obtain from RSS. The RSS scheme, which involves performing energy minimisation of random structures, is rather similar to the direct enumeration method ${ }^{44}$ that can be used to find the basin of attraction of granular or glassy systems, ${ }^{44-46}$ although RSS is somewhat more complicated as variable cells with different numbers of atoms are used (see Methods). The entropy associated with the (dimensionless) volume $v$ of a basin of attraction, $S=k_{\mathrm{B}} \ln v$, has been of considerable recent interest. ${ }^{47}$ It would therefore be intriguing to explore whether in this case, we can find an estimate for the basin volume and whether the corresponding basin entropy is related to the thermodynamic entropy that enters the Gibbs energy.

To estimate the entropy of the basin of the crystal structures found in RSS, we assume that (i) $S_{\mathrm{b}}$ of a crystal structure is extensive with respect to its system size, $S_{\mathrm{b}}=n s_{\mathrm{b}}$, where $n$ is the number of $\mathrm{TiO}_{2}$ formula units; and (ii) when performing energy minimisation with the same number of $\mathrm{TiO}_{2}$ formula units, $n s_{\mathrm{b}}$ is proportional to the logarithm of the frequency of finding a certain crystal structure. With these strong assumptions, we are able to infer the relative basin entropy for each crystal structure considered. The value of $s_{\mathrm{b}}$ obtained in this way depends on the frequency of occurrence of each structure for a given choice of $n$. However, for structures whose unit cells do not have compatible numbers of formula units, we can nevertheless infer the difference in $s_{\mathrm{b}}$ between two such polymorphs provided that they are found in searches together with other structures that have a well-defined $s_{\mathrm{b}}$ for both values of $n$.
To test our assumptions, and to compare the basin entropy $s_{\mathrm{b}}$ of the crystal structures and the thermodynamic entropy $S$, we plot them against each other in Fig. 2. Given that the two sets of entropies were estimated using completely different approaches, it is remarkable how similar their spread is. The Pearson correlation coefficient (PCC) is estimated to be $r \approx 0.3$, and the root mean squared error (RMSE) is $0.2 k_{\mathrm{B}}$. The value of the PCC may at first glance seem low, but note that the entropy difference between different phases of this system is itself relatively small, so even a rather small RMSE can significantly reduce the PCC. Moreover, we can notice that low enthalpy structures tend to have $s_{\mathrm{b}}$ larger than $S$ : low enthalpy phases are found more frequently in RSS than one might expect from their thermodynamic entropies. This may be because the way the initial structures are prepared and the subsequent enthalpy minimisation is performed during RSS can introduce a bias towards locating deeper minima, which is advantageous if one aims to find stable phases over high energy ones. The trend of finding more frequently the low enthalpy structures has been observed in a previous study that also involves sampling polymorphs of ionic solids. ${ }^{48}$ To achieve a better estimate of $S$, one can thus use a linear combination such as $s_{\mathrm{b}}-a H$, where $a$ is a parameter to the fit $a H+$ constant $=s_{\mathrm{b}}-S$ over all data points. We show a comparison between this adjusted entropy $s_{\mathrm{b}}-a H$ and $S$ in Fig. S4 of the ESI, $\dagger$ which shows a much stronger correlation $(r \approx 0.7$; see Section S4, ESI $\dagger)$. Of course, determining the value of $a$ requires prior knowledge of $S$, so such a correction scheme is less useful in practice.

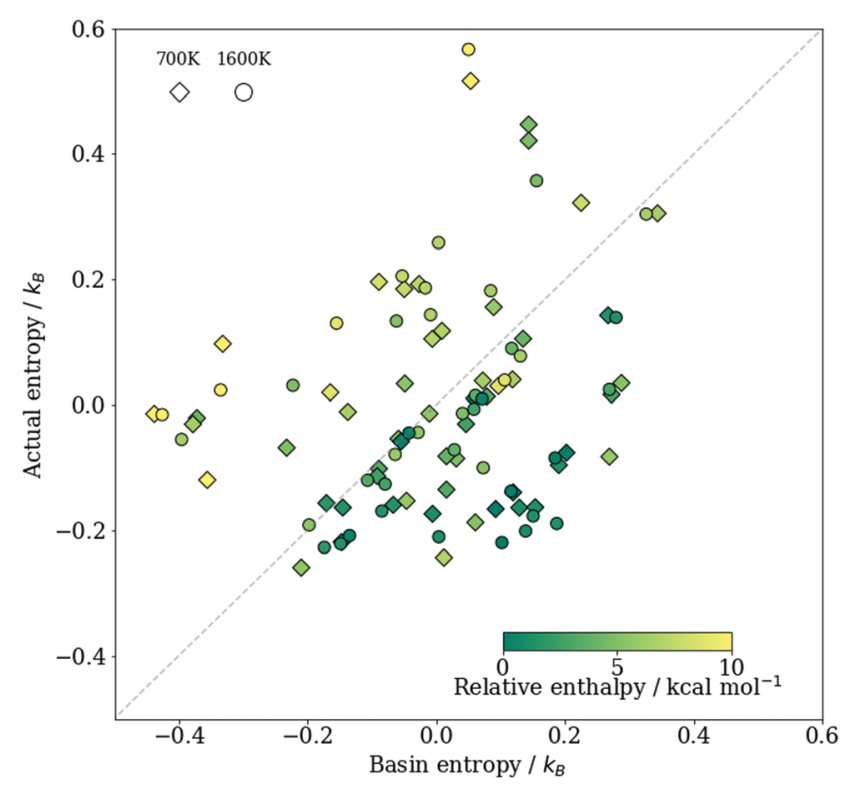

Fig. 2 A comparison between the basin entropy $s_{\mathrm{b}}=k_{\mathrm{B}} \ln (v) / n$ and the thermodynamic entropy $S=(H-G) / T$ per formula unit of $\mathrm{TiO}_{2}$. Diamonds show results at $700 \mathrm{~K}$ and circles show results at $1600 \mathrm{~K}$. Results obtained at all four pressures considered are all included, and the label of each point can be found in the ESI $\dagger$ (Fig. S4). Each data point is coloured according to the relative enthalpy of a structure compared to the ground state at the same pressure. As only the relative entropy under a given thermodynamic condition between different phases matters, we centred the data about the origin. 
Nevertheless, the results here demonstrate that the frequency of finding a structure in RSS does encode some information about its entropy. Furthermore, the correlation between $s_{\mathrm{b}}-S$ and $H$ means that the difference in $s_{\mathrm{b}}$ is a better estimator of the actual entropy difference between structures with similar enthalpies than for pairs with large enthalpy differences. Since we are typically interested in phases in the vicinity of the most favourable structure, this may be sufficient.

In order to demonstrate the level of approximation one can achieve in estimating the free energy using only RSS results, whenever we discuss ML-based approximations of $G$, we use $s_{\mathrm{b}}$ to estimate the true entropy. To obtain a more accurate estimate of $S$, one can use the harmonic approximation, ${ }^{6}$ or if resources allow, the TI method. ${ }^{6,8,9}$

\section{Machine-learning prediction of enthalpy and entropy}

As the number of local minima in the PES grows exponentially with increasing system size, it is difficult to ensure that all the important structures have been found during RSS. Indeed, not all the previously characterised or new structures of have been found at each pressure considered. There may also be situations where a certain crystal structure is known for an analogous system, but may not have been considered for the system of interest. In all such cases, we may wish to predict the enthalpies as well as the free energies of such structures. Of course, for a certain lattice arrangement, one can choose to perform energy minimisations and free-energy calculations, but it would be convenient to have a surrogate model that can quickly assess its enthalpy and basin entropy.

We developed such an approximate model using the kernel ridge regression (KRR) approach. In the following, we only discuss estimating $H$; the estimation of $S_{\mathrm{b}}$ follows the same workflow. For a given pressure, an enthalpy estimate function defined on a given structure $A$ is represented as a linear sum over kernel functions

$$
H^{\mathrm{ML}}(A)=\sum_{B \in \mathrm{M}} w(B) K(A, B),
$$

which are summed over a representative set of structures $\mathbf{M}$. The $\mathbf{M}$ representative structures are selected from the total $\mathrm{N}$ structures that are generated from RSS at each pressure. For this selection, we exploited the farthest-point sampling (FPS) approach, which is a greedy algorithm that aims to select reference structures that are as diverse as possible by successively selecting a new point that is farthest from the ones already selected. The set of weights $\left\{w_{\mathrm{M}}\right\}$ can be determined using

$$
w_{\mathrm{M}}=\left(K_{\mathrm{MM}}+K_{\mathrm{MN}} \Lambda^{-1} K_{\mathrm{MN}}^{\mathrm{T}}\right)^{-1} K_{\mathrm{MN}} \Lambda^{-1} H_{\mathrm{N}},
$$

where $K_{\mathrm{MM}}, K_{\mathrm{NN}}, K_{\mathrm{MN}}$ denote the kernel matrix between the $\mathrm{M}$ representative set, between the $\mathrm{N}$ complete set, and between the representative and the complete set, respectively; $\Lambda$ is an $N \times N$ diagonal matrix that is commonly used in the KRR framework to regularise the fit; and $H_{\mathrm{N}}$ is the enthalpy per formula unit of the N structures found by RSS. To estimate the statistical error due to the finite size of the training set $\mathrm{N}$, we employed a subsampling technique: ${ }^{49}$ we created an ensemble of KRR models using a subset of the training data and used the variance of these model predictions to infer the uncertainties. More details of the KRR model can be found in the ESI. $\dagger$

We plot the predictions of the KRR model alongside values obtained in direct energy minimisation simulations in the ESI $\dagger$ [Fig. S2]. The agreement of the prediction with the calculated enthalpies is excellent, demonstrating that the ML model is able to capture the fundamentals of the interactions based on local environments, even though long-range coulombic terms are present in the empirical potential. The ML predictions for the basin entropy are less good, perhaps due to the intrinsic errors in the estimation of $s_{\mathrm{b}}$ for systems of small sizes, or because descriptions of equilibrium configurations cannot fully capture the basin volume. We note that representations other than the average SOAP kernel used here, as well as a different choice of the regression, can also be used for the predictions. ${ }^{50-52}$

\section{Phase behaviour and phase diagram estimation}

In Fig. 3(a), we show chemical potentials (i.e. Gibbs energies per formula unit) computed using TI at a relatively high temperature of $1600 \mathrm{~K}$. We chose this temperature because entropic effects have become important, but the majority of the phases of interest are still metastable across the pressure range considered. ${ }^{53}$ The baddeleyite phase and several of the newly considered phases [namely 0 -c- $I 4_{1} / a, 0$-e- $C 2 / c, 60$-c- $P c$ and 60-e- $\left.P 2_{1} / m\right]$ do not appear in Fig. 3(a), because they are no longer metastable at such high temperatures. Baddeleyite, for example, spontaneously converts into pyrite at approximately $1150 \mathrm{~K}$ when heated at $20 \mathrm{GPa}$. One particularly interesting transition is that between rutile and the new phase 40-aPnnm (see ESI $\dagger$ (Fig. S1)), which are structurally particularly similar. The transition between them appears to be continuous as the system is pressurised: while the space group changes, there does not seem to be any change in density or enthalpy. In a sense, the 40-a-Pnnm polymorph is thus merely a high-pressure analogue of the rutile phase. Intriguingly, Fig. 3(a) shows a new phase, $60-\mathrm{a}-R \overline{3}$, is the most stable phase above $\sim 60 \mathrm{GPa}$, even though for some of this pressure regime, its enthalpy is not the lowest. This example highlights the importance of properly accounting for the entropy of the polymorphs. A number of other new phases also have free energies lower than some of the known structures of, demonstrating the power of the RSS approach in successfully locating possible candidate phases for consideration.

To circumvent extensive and laborious free-energy calculations, we can approximate the chemical potential using solely the data obtained from RSS. If a structure is found in RSS at a particular pressure, we use $h(0 \mathrm{~K})-T s_{\mathrm{b}}$ to approximate its chemical potential, where $h$ is the enthalpy per formula unit. For structures that are not found, we first use the ML schemes outlined in Section IIIC to estimate the enthalpy and basin entropy, and then estimate the chemical potential using the same expression. These chemical potentials are plotted in Fig. 3(b); phases found in RSS are indicated by solid lines and phases not found by dashed lines. As RSS was performed at four pressures, linear interpolations were used between the intervals. Alternatively, if one has data at only one pressure, 
(a) free-energy calculation

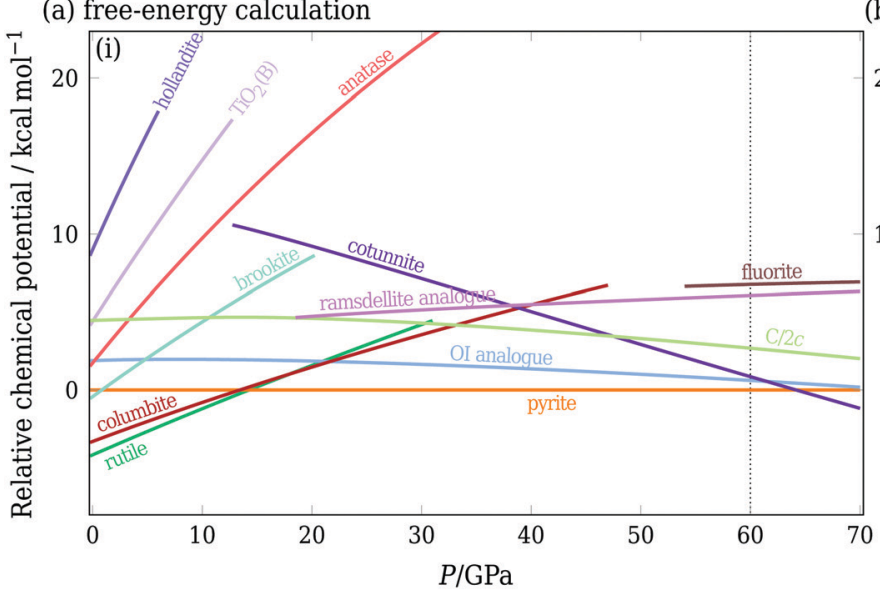

(b) ML toolkit approximation

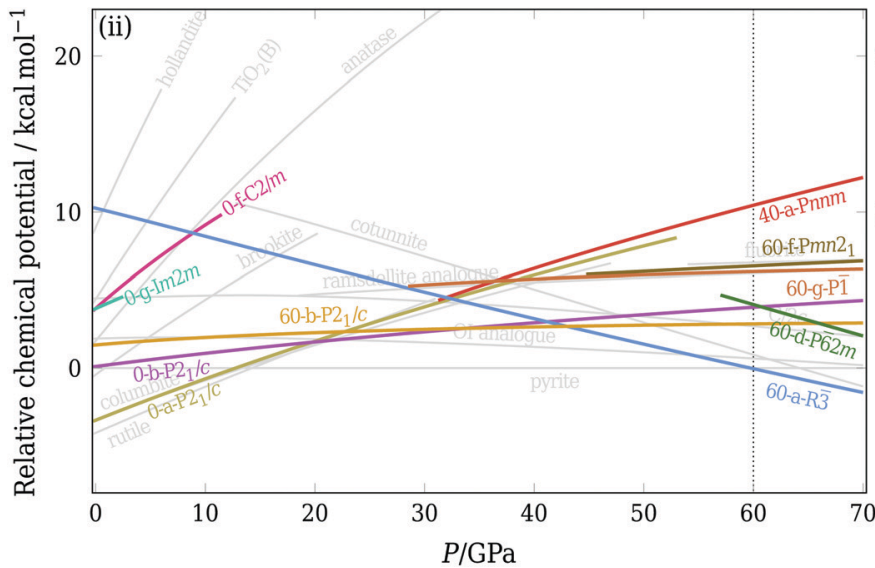

Fig. 3 Chemical potential at $1600 \mathrm{~K}$ expressed per formula unit and relative to the pyrite phase. Left panel: Results obtained using free-energy calculations. Right panel: Predictions using solely data obtained from RSS. If a structure is found during RSS at a given pressure, solid lines show the approximate chemical potential based on its enthalpy and basin entropy. If a structure is not found, dashed lines indicate the machine-learning prediction as described in Section IIIC. In each case, in panel (i), only the previously known phases are shown, and in panel (ii), new phases obtained from RSS are shown, with the structures from panel (i) repeated in grey. In (b), the error bar of potentially competitive phases is shown as a coloured band, and where a phase was not obtained at a given pressure from a random search, the marker is hollow and connected to other points by a dashed line. Note that the pressure axis covers different ranges in (a) and (b); dotted lines in (a) indicate the range of panel (b).

a simple linear approximation $G(P+\Delta P) \approx G(P)+\Delta P V$ for extrapolation to nearby pressures can be considered. ${ }^{2}$

The agreement with the calculations of Fig. $3(\mathrm{a})$ is rather remarkable, with broad consistency of both the shape of the curves and the ordering of the structures (within error bars). In part, this is because of the excellent prediction of the enthalpy (ESI, $\dagger$ Fig. S2); in addition, we note that the approximate approach is also able to account for example for the fact that the OI phase has a low enthalpy, but a narrow basin of attraction, and becomes less favourable at sufficiently high temperatures.

We note that Fig. 3 encodes the information required for constructing phase diagrams: the phase with the lowest chemical potential at a given pressure is the most stable for this potential at this temperature, and the crossover of the chemical potential curves is a point of phase coexistence. Using either the full freeenergy calculation approach or the ML-based approximation, we can repeat the procedure at several temperatures and can thus determine the phase diagram of this system [see ESI, $\dagger$ Fig. S7]. However, it ought to be borne in mind that a phase diagram hides much of the underlying thermodynamic behaviour, particularly for metastable phases, so the prediction of chemical potentials, as illustrated in Fig. 3(b), is thus a rather more rigorous test of the ML approach. Furthermore, since the free energies of many of the phases considered are similar to one another and many phases are metastable under certain conditions, predicting the metastabilities of all relevant polymorphs is particularly important.

\section{Conclusions}

This paper presents a framework that uses a combination of ML methods to help extract information from random crystal structure searches. This framework allows automatic classification and characterisation of possible solid phases, as well as providing an estimate of the phase diagram and metastabilities of solid polymorphs. The framework does not use any prior knowledge of the phase behaviour of the system and instead relies only on data obtained directly from a random structure search. Starting from the thousands of structures obtained from RSS, we first employ a pattern recognition approach to 
cluster similar structures with little manual input. We then estimate the basin entropy of the crystal structures found by assuming that their basin of attraction is related to the number of times each structure is found. Moreover, we use a machine learning approach to predict the enthalpy of unknown structures based on ones that are observed. Combining all these, we can estimate the free energies of solid phases and hence the thermodynamic phase behaviour of a system of interest with relatively little computational effort.

We tested the framework on the rather complicated system of $\mathrm{TiO}_{2}$ at ambient and high pressures. We predicted a number of possible phases and estimated their chemical potentials at finite temperatures. Even though we only explicitly showed the chemical potentials at a moderately high temperature, the framework that we present can be used for any reasonable temperature. Pair potentials similar to the ones used for our RSS are widely used for other binary oxides, ${ }^{54,55}$ so the new phases we found and the methodology introduced may be relevant to other oxides as well.

The methods we propose are transferable to other systems with many solid polymorphs, such as high-pressure solid hydrogen, ${ }^{56}$ perovskites, ${ }^{57}$ different phases of water-ice ${ }^{25}$ and molecular crystals. ${ }^{58} \mathrm{~A}$ useful strategy for predicting the phase diagram is first to use the framework to select competitive polymorphs using the data generated from crystal structure searches, and then to compute explicit free energies only of those phases. Such an approach would make the determination of phase behaviour of systems exhibiting many solid polymorphs using accurate free-energy calculations considerably more straightforward and computationally tractable.

\section{Conflicts of interest}

The authors declare no competing interests.

\section{Acknowledgements}

We acknowledge fruitful discussions with Daan Frenkel. We thank Michele Ceriotti, Stephen J. Cox, Edgar Engel and Bonan Zhu for critically reading the manuscript and providing useful feedback. BC acknowledges funding from the Swiss National Science Foundation (Project P2ELP2-184408). AR and BC acknowledge resources provided by the Cambridge Tier-2 system operated by the University of Cambridge Research Computing Service funded by EPSRC Tier-2 capital grant EP/P020259/1. CJP acknowledges financial support from the Engineering and Physical Sciences Research Council [Grant EP/P022596/1] and a Royal Society Wolfson Research Merit award.

\section{References}

1 S. M. Woodley and R. Catlow, Crystal structure prediction from first principles, Nat. Mater., 2008, 7, 937-946.
2 C. J. Pickard and R. Needs, Ab initio random structure searching, J. Phys.: Condens. Matter, 2011, 23, 053201.

3 Y. Wang, J. Lv, L. Zhu and Y. Ma, Crystal structure prediction via particle-swarm optimization, Phys. Rev. B: Condens. Matter Mater. Phys., 2010, 82, 094116.

4 L. Filion, M. Marechal, B. van Oorschot, D. Pelt, F. Smallenburg and M. Dijkstra, Efficient method for predicting crystal structures at finite temperature: variable box shape simulations, Phys. Rev. Lett., 2009, 103, 188302.

5 D. J. Wales and H. A. Scheraga, Global optimization of clusters, crystals, and biomolecules, Science, 1999, 285, 1368-1372.

6 B. Cheng and M. Ceriotti, Computing the absolute Gibbs free energy in atomistic simulations: applications to defects in solids, Phys. Rev. B, 2018, 97, 054102.

7 Z. Fu, Y. Liang, S. Wang and Z. Zhong, Structural phase transition and mechanical properties of $\mathrm{TiO}_{2}$ under high pressure, Phys. Status Solidi B, 2013, 250, 2206-2214.

8 C. Vega, E. Sanz, J. L. F. Abascal and E. G. Noya, Determination of phase diagrams via computer simulation: methodology and applications to water, electrolytes and proteins, J. Phys.: Condens. Matter, 2008, 20, 153101.

9 A. Reinhardt, Phase behavior of empirical potentials of titanium dioxide, J. Chem. Phys., 2019, 151, 064505.

10 B. Monserrat, N. D. Drummond, P. Dalladay-Simpson, R. T. Howie, P. L. Ros, E. Gregoryanz, C. J. Pickard and R. J. Needs, Structure and metallicity of phase V of hydrogen, Phys. Rev. Lett., 2018, 120, 255701.

11 H. Dekura, T. Tsuchiya, Y. Kuwayama and J. Tsuchiya, Theoretical and experimental evidence for a new postcotunnite phase of titanium dioxide with significant optical absorption, Phys. Rev. Lett., 2011, 107, 045701.

12 L. S. Dubrovinsky, N. A. Dubrovinskaia, V. Swamy, J. Muscat, N. M. Harrison, R. Ahuja, B. Holm and B. Johansson, The hardest known oxide, Nature, 2001, 410, 653-654.

13 K. Mukai and I. Yamada, Columbite-type $\mathrm{TiO}_{2}$ as a negative electrode material for lithium-ion batteries, J. Electrochem. Soc., 2017, 164, A3590-A3594.

14 J. Staun Olsen, L. Gerward and J. Z. Jiang, On the rutile/$\mathrm{PbO}_{2}$-type phase boundary of $\mathrm{TiO}_{2}, J$. Phys. Chem. Solids, 1999, 60, 229-233.

15 M. Matsui and M. Akaogi, Molecular dynamics simulation of the structural and physical properties of the four polymorphs of $\mathrm{TiO}_{2}$, Mol. Simul., 1991, 6, 239-244.

16 C. J. Pickard and R. J. Needs, High-pressure phases of silane, Phys. Rev. Lett., 2006, 97, 045504.

17 C. J. Pickard and R. J. Needs, Ab initio random structure searching, J. Phys.: Condens. Matter, 2011, 23, 053201.

18 S. Plimpton, Fast parallel algorithms for short-range molecular dynamics, J. Comput. Phys., 1995, 117, 1-19.

19 D. Frenkel and A. J. C. Ladd, New Monte Carlo method to compute the free energy of arbitrary solids. Application to the fcc and hcp phases of hard spheres, J. Chem. Phys., 1984, 81, 3188-3193.

20 R. O. Jones and O. Gunnarsson, The density functional formalism, its applications and prospects, Rev. Mod. Phys., 1989, 61, 689-746. 
21 J. P. Perdew, K. Burke and M. Ernzerhof, Generalized gradient approximation made simple, Phys. Rev. Lett., 1996, 77, 3865-3868.

22 J. P. Perdew, A. Ruzsinszky, G. I. Csonka, O. A. Vydrov, G. E. Scuseria, L. A. Constantin, X. Zhou and K. Burke, Restoring the density-gradient expansion for exchange in solids and surfaces, Phys. Rev. Lett., 2008, 100, 136406.

23 S. J. Clark, M. D. Segall, C. J. Pickard, P. J. Hasnip, M. I. Probert, K. Refson and M. C. Payne, First principles methods using CASTEP, Z. Kristallogr. Cryst. Mater., 2005, 220, 567-570.

24 M. Ceriotti, G. A. Tribello and M. Parrinello, Simplifying the representation of complex free-energy landscapes using sketchmap, Proc. Natl. Acad. Sci. U. S. A., 2011, 108, 13023-13028.

25 E. A. Engel, A. Anelli, M. Ceriotti, C. J. Pickard and R. J. Needs, Mapping uncharted territory in ice from zeolite networks to ice structures, Nat. Commun., 2018, 9, 2173.

26 S. De, A. P. Bartók, G. Csányi and M. Ceriotti, Comparing molecules and solids across structural and alchemical space, Phys. Chem. Chem. Phys., 2016, 18, 13754-13769.

27 A. Anelli, E. A. Engel, C. J. Pickard and M. Ceriotti, Generalized convex hull construction for materials discovery, Phys. Rev. Mater., 2018, 2, 103804.

28 J. Mavračić, F. C. Mocanu, V. L. Deringer, G. Csányi and S. R. Elliott, Similarity between amorphous and crystalline phases: The case of $\mathrm{TiO}_{2}$, J. Phys. Chem. Lett., 2018, 9, 2985-2990.

29 A. P. Bartók, R. Kondor and G. Csányi, On representing chemical environments, Phys. Rev. B: Condens. Matter Mater. Phys., 2013, 87, 184115.

30 V. L. Deringer and G. Csányi, Machine learning based interatomic potential for amorphous carbon, Phys. Rev. B, 2017, 95, 094203.

31 W. J. Szlachta, A. P. Bartók and G. Csányi, Accuracy and transferability of Gaussian approximation potential models for tungsten, Phys. Rev. B: Condens. Matter Mater. Phys, 2014, 90, 104108.

32 A. P. Bartók, S. De, C. Poelking, N. Bernstein, J. Kermode, G. Csányi and M. Ceriotti, Machine learning unifies the modelling of materials and molecules, Sci. Adv., 2017, 3, e1701816.

33 F. Musil, S. De, J. Yang, J. E. Campbell, G. M. Day and M. Ceriotti, Machine learning for the structure-energyproperty landscapes of molecular crystals, Chem. Sci., 2018, 9, 1289-1300.

34 L. Himanen, M. O. J. Jäger, E. V. Morooka, F. Federici Canova, Y. S. Ranawat, D. Z. Gao, P. Rinke and A. S. Foster, DScribe: library of descriptors for machine learning in materials science, Comput. Phys. Commun., 2020, 247, 106949, DOI: 10.1016/j.cpc.2019.106949.

35 B. Schölkopf, A. Smola and K.-R. Müller, Nonlinear component analysis as a kernel eigenvalue problem, Neural Comput., 1998, 10, 1299-1319.

36 M. Ester, H.-P. Kriegel, J. Sander and X. Xu, A density-based algorithm for discovering clusters in large spatial databases with noise, in Proceedings of the Second International Conference on Knowledge Discovery and Data Mining, AAAI Press, 1996, pp. 226-231.
37 M. J. Lyle, C. J. Pickard and R. J. Needs, Prediction of 10-fold coordinated $\mathrm{TiO}_{2}$ and $\mathrm{SiO}_{2}$ structures at multimegabar pressures, Proc. Natl. Acad. Sci. U. S. A., 2015, 112, 6898-6901.

38 D. R. Collins and W. Smith, Evaluation of $\mathrm{TiO}_{2}$ force fields, Tech. Rep. DL-TR-96-001 (Council for the Central Laboratory of the Research Councils, 1996).

39 V. Swamy, J. D. Gale and L. S. Dubrovinsky, Atomistic simulation of the crystal structures and bulk moduli of $\mathrm{TiO}_{2}$ polymorphs, J. Phys. Chem. Solids, 2001, 62, 887-895.

40 J. Trail, B. Monserrat, P. L. Ros, R. Maezono and R. J. Needs, Quantum Monte Carlo study of the energetics of the rutile, anatase, brookite, and columbite $\mathrm{TiO}_{2}$ polymorphs, Phys. Rev. B, 2017, 95, 121108.

41 X. G. Ma, P. Liang, L. Miao, S. W. Bie, C. K. Zhang, L. Xu and J. J. Jiang, Pressure-induced phase transition and elastic properties of $\mathrm{TiO}_{2}$ polymorphs, Phys. Status Solidi B, 2009, 246, 2132-2139.

42 Z.-G. Mei, Y. Wang, S. Shang and Z.-K. Liu, First-principles study of the mechanical properties and phase stability of $\mathrm{TiO}_{2}$, Comput. Mater. Sci., 2014, 83, 114-119.

43 T. Zhu and S.-P. Gao, The stability, electronic structure, and optical property of $\mathrm{TiO}_{2}$ polymorphs, J. Phys. Chem. C, 2014, 118, 11385-11396.

44 N. Xu, J. Blawzdziewicz and C. S. O'Hern, Random close packing revisited: Ways to pack frictionless disks, Phys. Rev. E: Stat., Nonlinear, Soft Matter Phys., 2005, 71, 061306.

45 D. Asenjo, F. Paillusson and D. Frenkel, Numerical calculation of granular entropy, Phys. Rev. Lett., 2014, 112, 098002.

46 S. Martiniani, K. J. Schrenk, K. Ramola, B. Chakraborty and D. Frenkel, Numerical test of the Edwards conjecture shows that all packings are equally probable at jamming, Nat. Phys., 2017, 13, 848-851.

47 (a) S. Martiniani, K. J. Schrenk, J. D. Stevenson, D. J. Wales and D. Frenkel, Turning intractable counting into sampling: Computing the configurational entropy of three-dimensional jammed packings, Phys. Rev. E, 2016, 93, 012906; (b) A. Daza, A. Wagemakers, B. Georgeot, D. Guéry-Odelin and M. A. F. Sanjuán, Basin entropy: A new tool to analyze uncertainty in dynamical systems, Sci. Rep., 2016, 6, 31416.

48 V. Stevanović, Sampling polymorphs of ionic solids using random superlattices, Phys. Rev. Lett., 2016, 116, 075503.

49 F. Musil, M. J. Willatt, M. A. Langovoy and M. Ceriotti, Fast and accurate uncertainty estimation in chemical machine learning, J. Chem. Theory Comput., 2019, 15, 906-915.

50 F. A. Faber, L. Hutchison, B. Huang, J. Gilmer, S. S. Schoenholz, G. E. Dahl, O. Vinyals, S. Kearnes, P. F. Riley and O. A. Von Lilienfeld, Prediction errors of molecular machine learning models lower than hybrid DFT error, J. Chem. Theory Comput., 2017, 13, 5255-5264.

51 C. Chen, W. Ye, Y. Zuo, C. Zheng and S. P. Ong, Graph networks as a universal machine learning framework for molecules and crystals, Chem. Mater., 2019, 31, 3564-3572.

52 C. J. Bartel, S. L. Millican, A. M. Deml, J. R. Rumptz, W. Tumas, A. W. Weimer, S. Lany, V. Stevanović, C. B. Musgrave and A. M. Holder, Physical descriptor for the Gibbs energy 
of inorganic crystalline solids and temperature-dependent materials chemistry, Nat. Commun., 2018, 9, 4168, DOI: 10.1038/s41467-018-06682-4.

53 It should however be noted that at high temperatures, intrinsic defects may become important. ${ }^{59,60}$ Some defects, such as Frenkel-style interstitials, can be accounted for with our approach, but the most important defects in $\mathrm{TiO}_{2}$ include charge transfer and cannot be modelled with simple empirical potentials. Given the large typical free energies of defect formation of all main types even at such high temperatures, ${ }^{59}$ it is not likely that they would significantly change the phase behaviour; however, an investigation of this behaviour with more advanced potentials would be particularly interesting.

54 G. V. Lewis and C. R. A. Catlow, Potential models for ionic oxides, J. Phys. C: Solid State Phys., 1985, 18, 1149-1161.

55 F. Shimojo, T. Okabe, F. Tachibana, M. Kobayashi and H. Okazaki, Molecular dynamics studies of yttria stabilized zirconia. I. Structure and oxygen diffusion, J. Phys. Soc. Jpn, 1992, 61, 2848-2857.

56 B. Cheng, G. Mazzola and M. Ceriotti, Evidence for supercritical behavior of high-pressure liquid hydrogen, 2019, arXiv preprint arXiv:1906.03341.

57 M. Murakami, K. Hirose, K. Kawamura, N. Sata and Y. Ohishi, Post-perovskite phase transition in $\mathrm{MgSiO}_{3}$, Science, 2004, 304, 855-858.

58 G. J. O. Beran, Modeling polymorphic molecular crystals with electronic structure theory, Chem. Rev., 2016, 116, 5567-5613.

59 J. Nowotny, M. A. Alim, T. Bak, M. A. Idris, M. Ionescu, K. Prince, M. Z. Sahdan, K. Sopian, M. A. Mat Teridi and W. Sigmund, Defect chemistry and defect engineering of $\mathrm{TiO}_{2}$-based semiconductors for solar energy conversion, Chem. Soc. Rev., 2015, 44, 8424-8442.

60 A. Sarkar and G. G. Khan, The formation and detection techniques of oxygen vacancies in titanium oxide-based nanostructures, Nanoscale, 2019, 11, 3414-3444. 